Proc. Indian Acad. Sci. (Chem. Sci.), Vol. 115, No. 4, August 2003, pp 307-317

(C) Indian Academy of Sciences

\title{
Kinetics of proton transfer in a green fluorescent protein: A laser-induced $\mathrm{pH}$ jump study
}

\author{
ROOP MALLIK ${ }^{1,3}$, JAYANT B UDGAONKAR ${ }^{1}$ and \\ G KRISHNAMOORTHY ${ }^{2, *}$ \\ ${ }^{1}$ National Centre for Biological Sciences, Tata Institute of Fundamental \\ Research, GKVK Campus, Bangalore 560 065, India \\ ${ }^{2}$ Department of Chemical Sciences, Tata Institute of Fundamental Research, \\ Mumbai 400 005, India \\ ${ }^{3}$ Present address: Department of Developmental \& Cell Biology, 2302 Natural \\ Sciences 1, University of California Irvine, Irvine, CA 92697, USA \\ e-mail:rmallik@uci.edu; jayant@ncbs.res.in; gk@tifr.res.in
}

MS received 30 May 2003

\begin{abstract}
The sub-millisecond protonation dynamics of the chromophore in S65T mutant form of the green fluorescent protein (GFP) was tracked after a rapid $\mathrm{pH}$ jump following laser-induced proton release from the caged photolabile compound $o$-nitrobenzaldehyde. Following a jump in $\mathrm{pH}$ from 8 to 5 (which is achieved within $2 \mu \mathrm{s}$ ), the fluorescence of S65T GFP decreased as a single exponential with a time constant of $\sim 90 \mu \mathrm{s}$. This decay is interpreted as the conversion of the deprotonated fluorescent GFP chromophore to a protonated non-fluorescent species. The protonation kinetics showed dependence on the bulk viscosity of the solvent, and therefore implicates bulk solvent-controlled protein dynamics in the protonation process. The protonation is proposed to be a sequential process involving two steps: (a) proton transfer from solvent to the chromophore, and (b) internal structural rearrangements to stabilize a protonated chromophore. The possible implications of these observations to protein dynamics in general is discussed.
\end{abstract}

Keywords. Green fluorescent protein; proton transfer; pH-jump; caged proton; protein dynamics.

\section{Introduction}

The intimate dependence between protein conformational dynamics and protein function is now widely appreciated. ${ }^{1-3}$ Estimating the level of the correlation between dynamics and function is the next step in functional biology. Time-domain experiments are most appropriate to quantify this correlation because static snapshots are blind to functionally important intermediate protein conformations. Using solution-based NMR spectroscopy, ${ }^{2,3}$ picosecond to millisecond scale fluctuations in conformation have been related ${ }^{4}$ recently to protein-protein interactions in the bacterial response regulator SpoOF. NMR measurements have provided insight into the way HIV1 protease $^{5}$ and calmodulin ${ }^{6}$ use conformational changes to achieve function. Proton pumps such as bacteriorhodopsin, ${ }^{7,8}$

*For correspondence 
ATP synthase ${ }^{9}$ and cytochrome oxidase ${ }^{10,11}$ rely upon conformational dynamics for their function, viz. proton transfer across the protein matrix.

The experimental difficulties which prevent quantification of functionally-relevant dynamics are: (i) identifying a usually weak signal corresponding to the motion of specific side-chains in the protein interior, (ii) relating it to a measurable functional property of the molecule, and (iii) isolating this signal from noise corresponding to functionally irrelevant motion and measuring it with sufficient temporal resolution. In addition, it has been difficult ${ }^{12,13}$ to reliably characterize motion on the critically important microsecond time-scale by the traditional techniques of NMR spectroscopy. We show here that green fluorescent protein ${ }^{14}$ (GFP), a very commonly used fluorescent marker in cell biology,${ }^{15}$ is a unique model system where all the above difficulties can be overcome. The functional property of GFP, i.e. its green fluorescence and sensitivity to $\mathrm{pH}$, can be used as a direct reporter of microsecond protein dynamics. This presents a unique opportunity to correlate function and dynamics directly at the level of local protein structure.

GFP consists of a compact cylinder made up of $\beta$-sheet strands and an $\alpha$-helix running along the axis of the cylinder..$^{15-17,19,20}$ The GFP chromophore, which gives the protein its characteristic green fluorescence, is well-protected in a hydrophobic region inside this cylinder, and is formed from residues Ser65-Tyr66-Gly67 (figure 1). The Tyr66 hydroxyl points towards the inner surface of the cylinder, and is almost perpendicular to the cylinder axis ${ }^{16}$. Wild-type (WT) GFP shows a complex excitation spectrum ${ }^{15}$ with excitation peaks around 395 and $480 \mathrm{~nm}$. Excitations at both these wavelengths give approximately similar emission profiles peaking at $508 \mathrm{~nm}$. The excitation spectrum is understood $^{15,18}$ in terms of existence of the Tyr66 hydroxyl group in protonated or deprotonated forms absorbing around 395 and $480 \mathrm{~nm}$ respectively. A complex hydrogen-bonding network near the GFP chromophore ${ }^{16,19}$ determines the protonation state of Tyr66, and hence the relative fluorescence excitation cross-sections of the two bands. The highly directional nature of hydrogen bonds implies that the excitation crosssection, and hence GFP fluorescence intensity, is extremely sensitive to local perturbations in structure near the GFP chromophore. ${ }^{19}$ Given this unique sensitivity of an easily observable functional property (i.e. fluorescence) to local structure, GFP is an ideal system to probe for functional dynamics.

The $\mathrm{pH}$ sensitivity of GFP fluorescence ${ }^{20-22}$ provides an experimental handle to visualize the dynamics around the chromophore. Upon excitation at $480 \mathrm{~nm}, \mathrm{pH}$ titration of the fluorescence intensity shows a sharp decrease on acidification, even in the $\mathrm{pH}$ range where the protein is in its native configuration. ${ }^{20-22}$ The pH-dependent crystal structure $^{20}$ of GFP (figure 1) indicates that protonation-deprotonation reaction of Tyr66 is associated with subtle variations in the orientation of a few side chains near the chromophore. Indeed, fluorescence correlation spectroscopy (FCS) measurements ${ }^{21}$ at $\mathrm{pH}$ values between 8 and 5 reveal that reversible proton transfer occurs between the chromophore and bulk solvent.

In the present work, we have used a laser-induced $\mathrm{pH}$ jump technique ${ }^{23-25}$ to monitor directly the kinetics of proton movement from bulk solvent to the GFP chromophore. We have chosen the S65T mutant form ${ }^{16}$ of GFP, where Ser65 has been replaced by the larger Thr group, because of an enhanced $\mathrm{pH}$ sensitivity of fluorescence ${ }^{20}$ and existence of only a single deprotonated species of the chromophore at neutral $\mathrm{pH}$ in this mutant form. ${ }^{19}$ The deprotonated chromophore is stabilized ${ }^{19,20}$ through $\mathrm{H}$-bonds donated by nearby polar groups such as Thr203, His148 and an ordered water molecule. Thus, S65T 

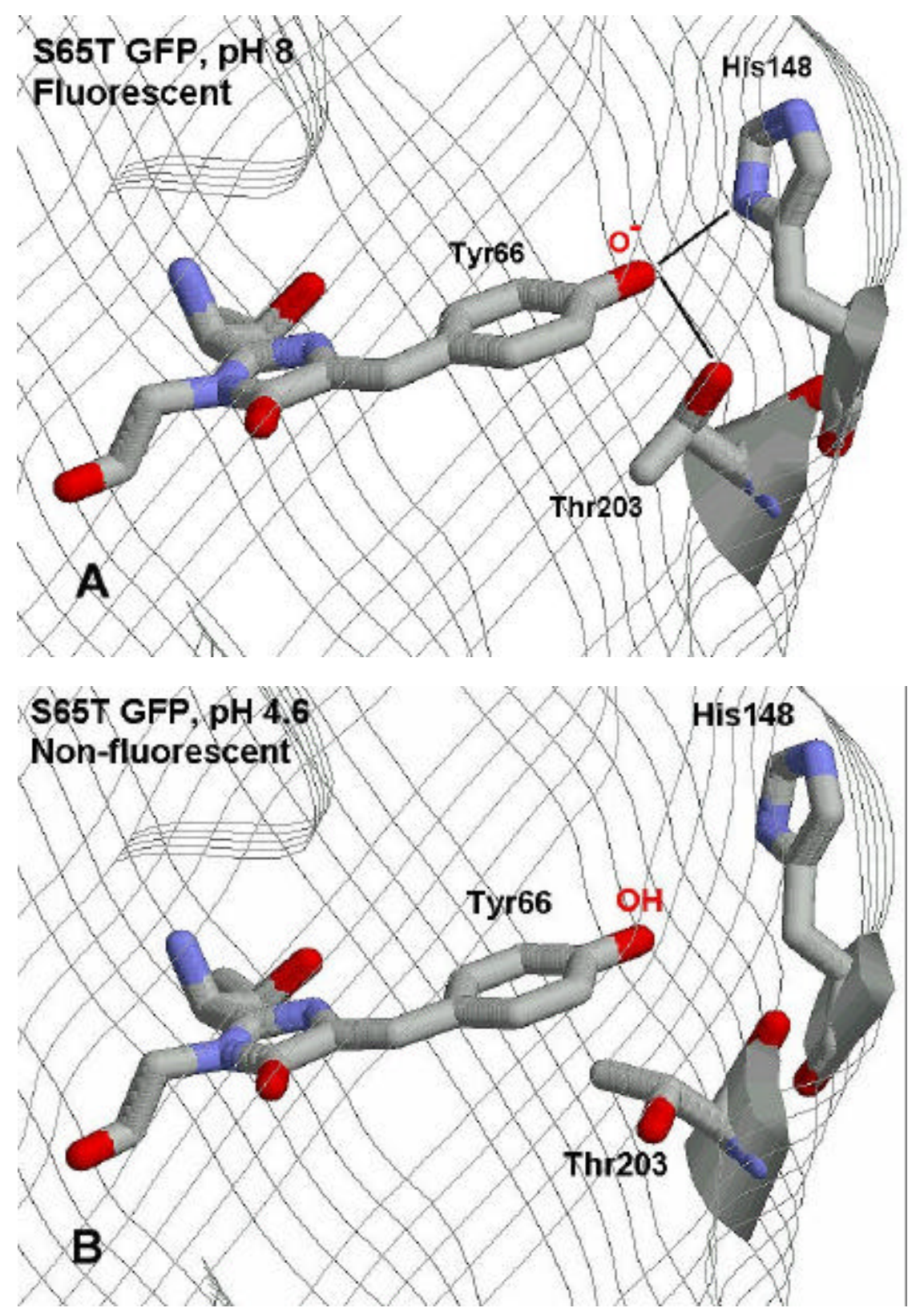

Figure 1. Structural changes in S65T GFP accompanying a change in $\mathrm{pH}$. (a) At pH 8 , a negative charge is stabilized on Tyr66 group of the chromophore through H-bonds (shown as thick lines) donated by Thr203 and His148. (b) Upon lowering the $\mathrm{pH}$, Thr203 rotates by $100^{\circ}$ and His 148 moves away slightly from the chromophore, ${ }^{20}$ breaking the connecting H-bonds. The cylindrical $\beta$-sheet structure of GFP was made using RASMOL. 
GFP has a single excitation peak around $480 \mathrm{~nm}$ corresponding to the deprotonated chromophore, and emits around $508 \mathrm{~nm}$. In wt GFP, excitation at $395 \mathrm{~nm}$ leads to excited state proton transfer ${ }^{26}$ to a nearby side chain and the usual emission at $508 \mathrm{~nm}$. This is not possible for S65T GFP due to the absence of a proton acceptor site. ${ }^{19}$ Thus, the fluorescence properties of S65T GFP are less complex when compared to wt GFP. We also monitored the dependence of the rate of proton transfer to GFP chromophore on solvent viscosity and implicate protein dynamics in the control of proton transfer function.

\section{Materials and methods}

\subsection{Protein characterization}

S65T GFP was a kind gift from Professor A S Verkman of the University of California, San Francisco. Details of protein expression and the purification protocol are as published. $^{27}$

Absorbance, fluorescence excitation and emission characteristics were measured at $1 \mu \mathrm{M}$ S65T GFP concentration in solutions of varying $\mathrm{pH}$ between $\mathrm{pH} 8$ and $\mathrm{pH} 5$ in $50 \mathrm{mM}$ phosphate-citrate-borate buffer. The fluorescence spectra at $\mathrm{pH} 7$ were found to have a single excitation peak at $482 \mathrm{~nm}$ and an emission peak at $510 \mathrm{~nm}$. A p $K_{a}$ of 5.8 was determined from fluorescence titration (data not shown). The decrease in fluorescence intensity on acidification from $\mathrm{pH} 8$ to 5 was found to be $85 \%$ of the value at $\mathrm{pH}$ 8. To check reversibility of fluorescence as a function of $\mathrm{pH}$, a solution of S65T GFP at $\mathrm{pH} 8$ was diluted into a $\mathrm{pH} 5$ buffer and equilibrated for $1 \mathrm{~h}$ before being restored to $\mathrm{pH} 8$ again. Fluorescence excitation and emission spectra were recorded at different $\mathrm{pH}$ values with glycerol concentrations of 0,10 and $20 \% \mathrm{v} / \mathrm{v}$. There was no noticeable change in the spectra as a result of addition of glycerol.

\subsection{Rapid laser-induced $p H$ jump and fluorescence detection}

Laser-induced photoexcitation of a caged compound ${ }^{24,25}$ (e.g. $O$-nitrobenzaldehyde, $o$-NBA), which becomes a weak acid (o-nitrosobenzoic acid with $\mathrm{p} K_{a} \sim 4$ ) on photoexcitation, was used to rapidly increase proton concentration of solutions. Using this technique, absorbance and structural volume changes have been used to monitor the reactions of photo-detached protons with other chemical species. ${ }^{23,24,28}$ The time required for completion of the $\mathrm{pH}$ jump was found by monitoring the absorbance of bromocresol green at $632 \mathrm{~nm}$ (also see ref. 24). The absorbance change, and hence the $\mathrm{pH}$ jump was complete within $2 \mu \mathrm{s}$ of the laser flash. Our set-up (figure 2) measures fluorescence changes occurring in response to the rapid $\mathrm{pH}$ jump. A Quantel (France) Brilliant-B Q-switched Nd:YAG laser giving $5 \mathrm{~ns}$ pulses at $1064 \mathrm{~nm}$ was frequency-tripled to obtain a $355 \mathrm{~nm}$ ultraviolet (UV) beam with pulse energy of $200 \mathrm{~mJ}$. This UV beam was focused just before the cuvette containing the sample solution, and generated $\mathrm{pH}$ jumps in a cylindrical volume of $4 \mathrm{~mm}$ diameter and $2 \mathrm{~mm}$ length in the cuvette (the activated volume). Fluorescence excitation (from a deuterium lamp) was focused to $2 \mathrm{~mm}$ diameter and was incident at the centre of the activated volume in an anti-parallel but slightly offcollinear incidence to the laser. Thus, the fluorescence excitation volume (or probed volume) was fully contained within the activated region. The fluorescence signal after filtering was detected by a 9-stage model R928 (Hamamatsu, Japan) photomultiplier tube 
(PMT), and recorded using a $500 \mathrm{MHz}$ digital oscilloscope. Data acquisition was triggered by the UV laser shot through a photodiode; thus, the zero of time was determined by the incoming laser flash.

This instrument was calibrated by photoactivation of a solution containing $2 \mathrm{mM}$ $o$-NBA and $1 \mu \mathrm{M}$ of the fluorescent $\mathrm{pH}$ indicator fluorescein at $\mathrm{pH} 8$ (figure 3). The $\mathrm{pH}$ jump-induced decrease in fluorescence showed that robust and repeatable $\mathrm{pH}$ jumps from pH 8 to $\mathrm{pH} 5$ could be obtained. Diffusion from outside the activated region can equilibrate the $\mathrm{pH}$ change; however we found negligible $\mathrm{pH}$ drift at $\mathrm{pH} 5$ even on a timescale of several seconds (figure 3, inset). This implies that the probed volume was very well-contained inside the activated region, and thus homogeneous $\mathrm{pH}$ conditions existed in the probed volume for several seconds. The activating laser beam induced a broadband cuvette luminescence which can mask the fluorescence from GFP. With careful optical arrangement these effects were reduced such that they last for less than $20 \mu$ s after the passage of laser shot, as determined in a control experiment where the photomultiplier tube signal for laser incident on water was recorded. Keeping this in mind, we rejected data below $20 \mu \mathrm{s}$ in all results stated. For $\mathrm{pH}$ jump experiments with protein, no additional buffer was used. A $2 \mu \mathrm{M}$ concentration of S65T GFP in aqueous solution of $2 \mathrm{mM} o$-NBA was sufficient to stabilize the pre-pulse $\mathrm{pH}$ at $\mathrm{pH}$. In a control experiment using S65T GFP solution at $\mathrm{pH} 8$ with no $o$-NBA, no change in S65T GFP fluorescence intensity occurred upon a laser flash. Thus, the observed fluorescence decay was confirmed to be a $\mathrm{pH}$ jump-induced protonation reaction. This also confirmed that the laser does not induce photobleaching of the protein. For glycerol concentrations up to $20 \%(v / v)$, there was no noticeable change in the achieved magnitude of the $\mathrm{pH}$ jump. The structure (figure 1) of S65T GFP at pH 8 and 4.6 was drawn using RASMOL and the PDB files with accession codes 1EMG and 1C4F (www.rcsb.org/pdb/).

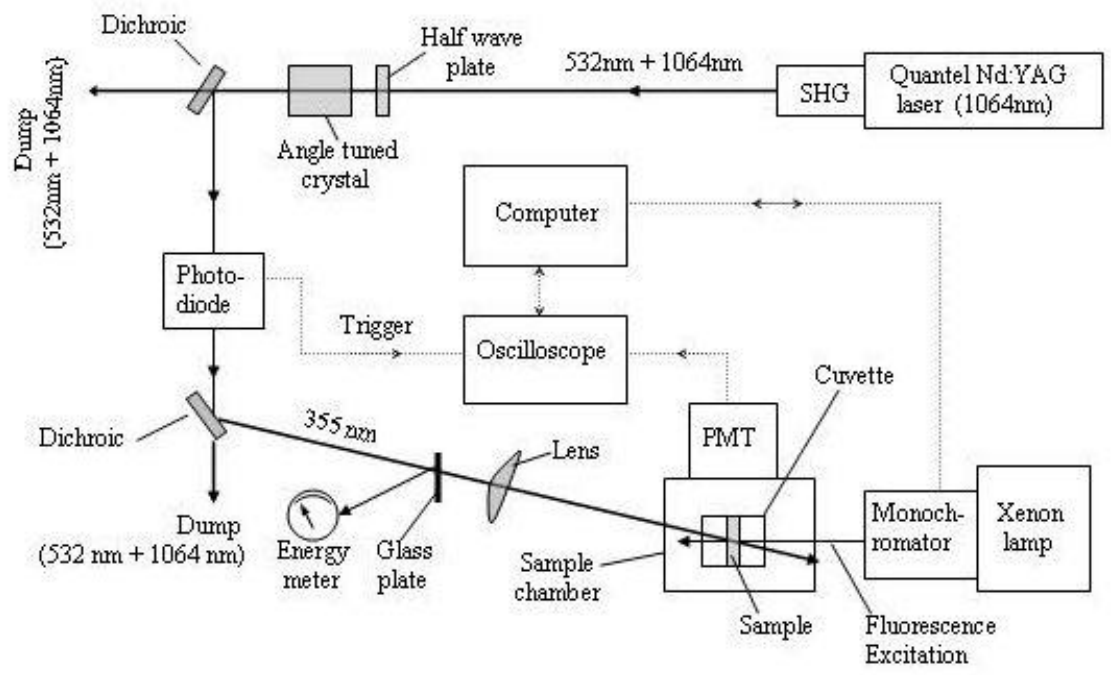

Figure 2. Schematic of experimental setup for carrying out laser-induced rapid $\mathrm{pH}$ jumps. SHG - second harmonic generator. PMT - photomultiplier tube. 


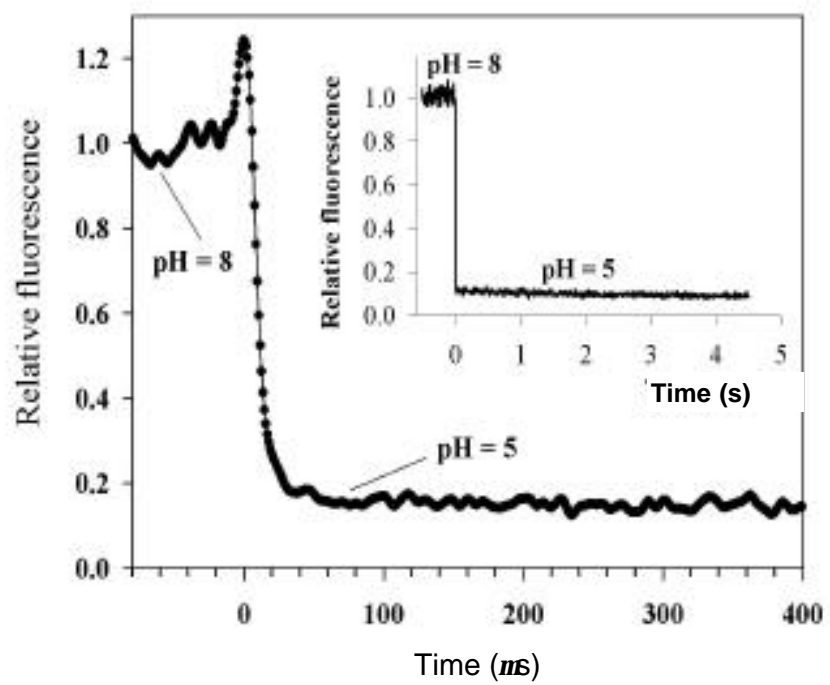

Figure 3. Relative fluorescence change (normalized to the fluorescence intensity at $\mathrm{pH} 8$ ) in a $1-\mu \mathrm{M}$ fluorescein solution after laser-induced $\mathrm{pH} 8$ to 5 jump. The $\mathrm{pH}$ jump was complete within $2 \mu \mathrm{s}$. The $o$-NBA concentration used was $2 \mathrm{mM}$. Sampling frequency for the digital oscilloscope was $2.5 \mathrm{MHz}$. Excitation: $480 \mathrm{~nm}$, emission: $510 \mathrm{~nm}$. Laser shot arrives at the zero of time. First $20 \mu \mathrm{s}$ of data are not reliable due to cuvette luminescence, which appears as a transient spike immediately after laser activation (see §2). The inset shows the relative fluorescence change in fluorescein up to longer time-scales.

\section{Results and discussion}

\subsection{Laser-induced $\mathrm{pH}$ jump}

The main aim of the present work is to measure directly the rate of proton flow from bulk solvent to the chromophore buried inside GFP by using a pH jump from $\mathrm{pH} 8$ to 5. S65T GFP has an extremely robust tertiary structure, and is in its native state even at $\mathrm{pH} 4.6$, as brought out by circular dichroism ${ }^{22}$ and crystal structure studies. ${ }^{20}$ Thus, the protein remains in its native state during the $\mathrm{pH}$ jump from 8 to 5 . The decrease in fluorescence intensity on lowering the $\mathrm{pH}$ (from $\mathrm{pH} 8$ to $\mathrm{pH}$ ) can therefore be interpreted as due to decrease in excitation cross-section at $480 \mathrm{~nm}$ caused by a single protonation reaction, which converts the deprotonated Tyr66 residue to a protonated form at low $\mathrm{pH}$. The following evidence reinforces this scenario: (i) $\mathrm{pH}$-dependent absorption spectra ${ }^{22}$ of S65T GFP show a single isobestic point, (ii) the fluorescence changes were reversible with $\mathrm{pH}$ - fluorescence intensity lost upon lowering the $\mathrm{pH}$ (data not shown) from 8 to 5 was found to recover to $98 \%$ upon restoring the solution to the initial $\mathrm{pH}$ of 8 (also see ref. 22), and (iii) autocorrelation curves for fluorescence fluctuation determined by FCS measurements ${ }^{21}$ show $\mathrm{pH}$-dependence of proton transfer rates, and this $\mathrm{pH}$-dependence disappears on replacement of Tyr66 by a non-protonable Trp residue.

Stopped-flow $\mathrm{pH}$ jump experiments from $\mathrm{pH} 8$ to 5 (data not shown) showed $85 \%$ decrease in fluorescence on lowering the $\mathrm{pH}$, which was complete within the dead time 
(3.5 ms) of the experiment (also see ref. 22). The magnitude of this fluorescence change matched $^{22}$ with that expected from a $\mathrm{pH}$ titration of S65T GFP. To capture the kinetics of fluorescence change, a faster experiment is required in which the $\mathrm{pH}$ can be lowered from $\mathrm{pH} 8$ to 5 , and the decrease in fluorescence observed thereafter. We have used the laserinduced $\mathrm{pH}$ jump technique $\mathrm{e}^{23-25}$ to investigate the kinetics of fluorescence changes in GFP resulting from the rapid release of protons in the bulk solvent. Basically the method involves rapid release of protons following photolysis of $o$-nitrobenzaldehyde. ${ }^{23,24,29}$

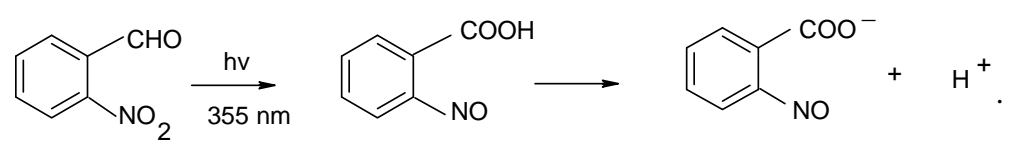

Subsequent to the laser ( $355 \mathrm{~nm}, 5 \mathrm{~ns}$ pulse width) pulse-induced generation of protons, the fluorescence intensity of GFP was monitored (see $\$ 2$ for more details).

The lowermost curve in figure 4 presents the sub-millisecond response of S65T GFP fluorescence to a laser photolysis-induced jump in $\mathrm{pH}$ from 8 to 5 . The relative fluorescence change (normalized to $\mathrm{pH} 8$ values) of S65T GFP could be fitted to a single exponential process with a time constant of $87( \pm 1) \mu \mathrm{s}$. An almost $85 \%$ decrease in the fluorescence intensity was seen. Considering that the magnitude of this change matched that seen in stopped flow experiments, and that those experiments showed no further change in fluorescence on longer timescales ${ }^{22}$ it is clear that the entire protonation process was captured. The time-scale of the fluorescence change $(\tau \sim 87 \mu \mathrm{s})$ is strikingly longer when compared to that of the protonation of fluorescein in aqueous solution (figure 3 ), where the entire process occurred within the detection resolution of $\sim 10 \mu \mathrm{s}$. Thus the observed process of $\sim 87 \mu \mathrm{s}$ in GFP shows that protonation of GFP chromophore is retarded by the protein matrix. Equilibrium FCS studies ${ }^{21}$ had reported a $\mathrm{pH}$-independent protonation process wherein the proton from the GFP chromophore is transferred to an internal acceptor. Furthermore, this process was proposed to be slower than the rate of protonation from the bulk solvent. ${ }^{21}$ However, there was no evidence for such a process in our experimental observations. Presence of such an internal proton acceptor, which is not in direct equilibrium with external $\mathrm{pH}^{21}$ could have resulted in appearance of a second kinetic phase with negative amplitude in our experiments, contrary to our observations (figure 4). This suggests that the $\mathrm{pH}$-independent internal proton transfer process is not functionally relevant for proton transfer between bulk solvent and chromophore.

\subsection{Protonation requires involvement of protein dynamics}

Since the rate of protonation of the GFP chromophore is significantly slowed down (figure 4) in comparison to that of a small molecule in solution (figure 3), it is reasonable to assume that the protein matrix and its dynamics control the rate of proton transfer between the chromophore and bulk solvent. $\mathrm{pH}$ jump experiments performed in viscous solutions (obtained by adding glycerol) showed a clear slowing down of the decay kinetics with increasing viscosity (figure 4). The kinetics could be fitted to single exponential decay for all viscosities investigated, and the time constants obtained were 87 $( \pm 1), 134( \pm 1), 193( \pm 2)$ and $264( \pm 3) \mu$ s at glycerol concentrations of $0,10,20$ and $30 \%$ $v / v$ respectively. The relative time constants (normalized to $0 \%$ glycerol value) so 
obtained have been plotted against relative viscosity (see figure 4, inset). A strong linear dependence of the time constant on viscosity was observed in the viscosity range investigated. (It should be mentioned that the process of $\mathrm{pH}$ jump-induced decrease in fluorescence of fluorescein (figure 3) occurred within the detection resolution of $\sim 10 \mu \mathrm{s}$ even at $30 \%$ glycerol (data not shown).) A weaker viscosity dependence of the time constant for protonation-deprotonation of Tyr66 was $\operatorname{seen}^{21}$ in FCS measurements on GFP, contrary to our measurements. Although the results obtained from an equilibrium fluctuation experiment such as FCS and a perturbation method such as pH-jump employed in the present investigation should provide similar results in principle, the differences in viscosity dependence could be due to insufficiency of modelling the FCS results and difficulties associated with resolving various kinetic processes in FCS. In contrast, the $\mathrm{pH}$-jump method employed in the present work offers a direct way to estimate the kinetics of the protonation process.

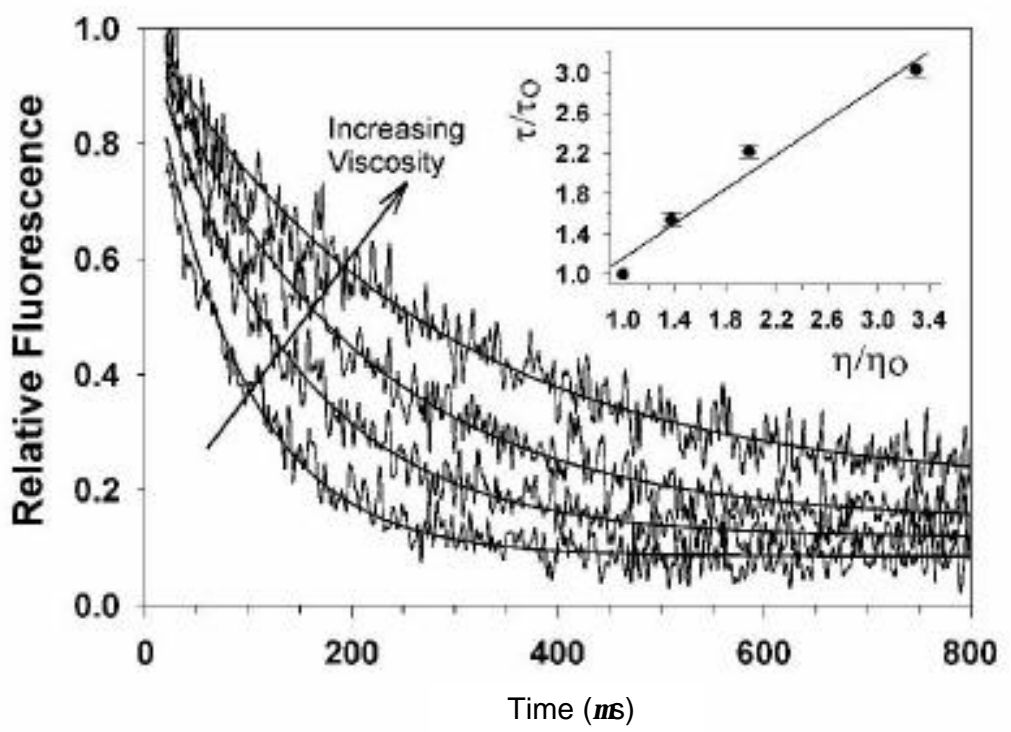

Figure 4. Kinetics of the fluorescence change in S65T GFP after laser-induced jumps in $\mathrm{pH}$ from 8 to 5 . Relative fluorescence intensity as a function of time is plotted for solutions of increasing viscosity, bottom to top: $0,10,20$ and $30 \%(v / v)$ of glycerol in solution. Each data set is an average of 3 recordings. Fluorescence excitation: $480 \mathrm{~nm}$, emission: 508 (FWHM 18.5) nm. Laser shot arrives at zero of time. The first $20 \mu \mathrm{s}$ of data after laser shot was rejected for reasons mentioned in text. Thick smooth lines are single exponential fits to respective (noisy) data sets. The time constants for fluorescence change are $87( \pm 1), 134( \pm 1), 193( \pm 2)$ and 264 $( \pm 3) \mu$ s at glycerol concentrations of $0,10,20$ and $30 \%(v / v)$ respectively. $2 \mu \mathrm{M}$ S65T GFP in an aqueous solution at initial $\mathrm{pH} 8$, and containing $2 \mathrm{mM} o$-NBA was used. Inset: Relative time constant $\tau / \tau_{0}\left(\tau_{0}=87 \mu \mathrm{s}\right.$, time constant for $0 \%$ glycerol solution) as a function of relative viscosity $\eta / \eta_{0}\left(\eta_{0}=\right.$ coefficient of viscosity for $0 \%$ glycerol solution). A straight line has been drawn by inspection to emphasize the linearity of the data. 
The observed viscosity dependence of protonation rates implies that chromophore protonation from bulk solvent requires the involvement of protein dynamics. Proton transfer mechanisms requiring protein dynamics have earlier been discussed for the proton pump mechanism of bacteriorhodop $\sin ^{9}$ and cytochome oxidase. ${ }^{10,11}$ Recently, the molecular mechanism for redox-driven proton transfer to a buried iron-sulphur cluster has been elucidated ${ }^{30}$ in Azotobacter vinelandii, where orientation and the $\mathrm{p} K_{a}$ of a single amino acid residue (Asp15) is found to be critical to proton flow across a complex proton pathway.

Crystal structures ${ }^{19,20}$ of S65T GFP at $\mathrm{pH} 8$ and 4.6 indicate likely candidates for the rate-determining step in the $\mathrm{pH}$ jump-induced protonation of the GFP chromophore. Two possible models can be visualized: Model $A$ - proton arrival at the chromophore site itself is rate-limiting, and structural rearrangements in the trans-matrix proton pathway determine protonation kinetics. Model $B$-Rearrangement of side-chains near the chromophore is rate-limiting, and determines the time-scale of response of fluorescence to a change in $\mathrm{pH}$ of the bulk solvent. Thus, according to this model, the rate of arrival of protons is not rate-limiting. Crystal structures ${ }^{19,20}$ suggest that proton entry to Tyr66 from solution can occur through a network of bound water molecules. A very small change in the position of these water molecules between $\mathrm{pH} 8$ and 4.6 is seen. ${ }^{20}$ Thus, according to model A, the observed kinetics of fluorescence decrease (figure 4) would represent the kinetics of proton movement from the protein-water interface to Tyr66 through a H-bonded network involving the bound water molecules. However, significant rearrangement of side chains around Tyr66 is required in the protonated form (figure 1b). Thr203 and His148 donate H-bonds to Tyr66 and stabilize an anionic fluorescent chromophore at pH 8 (see figure 1a). Tyr66 and His148 move away in the protonated form (figure $1 \mathrm{~b}$ ). Thus model $\mathrm{B}$ would demand that protons enter rapidly (though the $\mathrm{H}$ bonded network mentioned above) on lowering the $\mathrm{pH}$, but stabilization of the protonated Tyr66 occurs only after the reorientation of Tyr203 and His148. Movement of these side chains would then dictate the rate of protonation and subsequent decrease in fluorescence. The third scenario could be visualized as a concerted process of proton movement from the bulk to the phenolate group of the GFP chromophore. In this model, the time-scales of proton transfer through the H-bonded network, and the actual protonation process are not separable. Dynamic simulations of proton transfer from bulk solvent to the GFP chromophore, similar to those performed recently on internal proton transfer in GFP, ${ }^{31}$ and proton transfer in chains of water molecules, ${ }^{32,33}$ would be useful in resolving the questions raised above.

Irrespective of the actual mechanism of proton transfer from the bulk solvent to the GFP chromophore, our observation on the viscosity dependence of protonation kinetics (figure 4) shows clearly that the kinetics of protonation of GFP is controlled by bulk solvent viscosity. It is easy to visualize that movement of various side-chains would be involved in all the models described above. Control of conformational dynamics of native protein structures by solvent viscosity has been studied by a variety of techniques. ${ }^{34-36}$ Ligand binding, $\mathrm{H}$-exchange kinetics and diffusion of small molecules have been used as handles to infer the role of solvent viscosity in modulating protein dynamics. Kramer's theory of diffusional barrier crossing ${ }^{34,37,38}$ predicts the rate of diffusional barrier-crossing to be inversely proportional to viscosity in the high friction limit such as those encountered in processes in aqueous media. The linear dependence of the apparent rate of protonation (figure 4, inset) on viscosity could be explained by Kramer's theory. It should be pointed out that although the observed viscosity-dependence is in line with 
Kramer's theory, it does not enable us to differentiate between various models mentioned above. All the three models described above involve protein dynamics albeit at various levels, and hence could be controlled by solvent viscosity. Nevertheless our observation of viscosity-dependence clearly demonstrates the control of protein function by the dynamics of its constituent segments. Further, experiments aimed at identifying localized dynamics that control protonation kinetics are in progress.

In summary, an experiment which measures functionally important motion in the protonation-induced change in fluorescence of the S65T GFP chromophore has been described. The kinetics of the $\mathrm{pH}$-induced change in fluorescence is found to be viscosity-dependent, and therefore implicate protein dynamics in the proton transfer process.

\section{Acknowledgements}

This work was funded by the Tata Institute of Fundamental Research, and a grant from the Department of Science and Technology, Government of India to JBU.

\section{References}

1. Fenimore P W, Frauenfelder H, McMahon B H and Parak F G 2002 Proc. Natl. Acad. Sci. (USA) 9916047

2. Kay L E 1998 Nature Struct. Biol. (NMR Suppl.) 5513

3. Stock A 1999 Nature (London) 400221

4. Feher V A and Cavanagh J 1999 Nature (London) 400289

5. Nicholson L K, Yamazaki T, Torchia D A, Grzesiek S, Bax A, Stahl S J, Kaufman J D, Wingfield P T, Lam P Y and Jadhav P K 1995 Nature Struct. Biol. 2274

6. Lee A L, Kinnear S A and Wand A J 2000 Nature Struct. Biol. 772

7. Luecke H, Schobert B, Richter H T, Cartailler J P and Lanyi J K 1999 Science 286255

8. Subramaniam S and Henderson 2000 Nature (London) 406653

9. Abrahams J P, Leslie A G, Lutte R and Walker J E 1994 Nature (London) 370621

10. Iwata S, Ostermier C, Ludwig B and Michel H 1995 Nature (London) 376660

11. Tsukihara T, Aoyama H, Yamashita E, Tomizaki T, Yamaguchi H, Shinzawa-Itoh, Nakashima R, Yaono R and Yoshikawa S 1996 Science 2721136

12. Cavanagh J and Akke M 2000 Nature Struct. Biol. 711

13. Wand J A 2001 Nature Struct. Biol. 8926

14. Shimomura O, Johnson F H and Saiga Y 1962 J. Cell. Comp. Physiol. 59223

15. Tsien R Y 1998 Annu. Rev. Biochem. 67509

16. Ormo M, Cubitt A B, Kallio K, Gross L A, Tsien R Y and Remington S J 1996 Science 2731392

17. Yang F, Moss L G and Phillips G N Jr 1996 Nature Biotechnol. 141246

18. Heim R, Prasher D C and Tsien R Y 1994 Proc. Natl. Acad. Sci. (USA) 9112501

19. Brejc K, Sixma T K, Kitts P A, Kain S R, Tsien R Y, Ormo M and Remington S J 1997 Proc. Natl. Acad. Sci. (USA) 942306

20. Elsliger M, Wachter R M, Hanson G T, Kallio K and Remington S J 1999 Biochemistry 38 5296

21. Haupts U, Maiti S, Schwille P and Webb W W 1998 Proc. Natl. Acad. Sci. (USA) 95 13573

22. Kneen M, Farinas J, Li Y and Verkman A S 1998 Biophys. J. 741591

23. Viappiani C, Bonettu G, Carcelli M, Ferrari F and Sternieri A 1998 Rev. Sci. Instrum. 69 270

24. Abbruzzetti S, Crema E, Masino L, Veeli A, Viappiani C, Small J R, Libertini L J and Small E W 2000 Biophys. J. 78405

25. Barth A and Corriedaggo J E T 2002 Biophys. J. 832864

26. Chattoraj M, King B A, Bublitz G U and Boxer S G 1996 Proc. Natl. Acad. Sci. (USA) 93 8362 
27. Swaminathan R, Hoang C P and Verkman A S 1997 Biophys. J. 721900

28. Gutman M and Nachliel E 1990 Biochim. Biophys. Acta 1015391

29. George M V and Scaiano J C 1980 J. Phys. Chem. 84492

30. Chen K, Hirst J, Camba R, Bonagura C A, Stout C D, Burgess B K and Armstrong F A 2000 Nature (London) 405814

31. Lill M A and Helms V 2002 Proc. Natl. Acad. Sci. (USA) 992778

32. Pomes R and Roux B 1996 J. Phys. Chem. 1002519

33. Brewer M L, Schmitt U W and Voth G A 2001 Biophys. J. 801691

34. Gavish B and Yedgar S 1995 Protein-solvent interactions (ed.) R B Gregory (New York: Marcel Dekker) p. 343

35. Gregory R G, Rosenberg A, Knox D and Percy A J 1990 Biopolymers 291175

36. Ansari A, Lones C M, Henry E R, Hofrichter J and Eaton W A 1992 Science 2561796

37. Kramers H A 1940 Physica (Utrecht) 7284

38. Hanggi P, Talkner P and Borkovec M 1990 Rev. Mod. Phys. 62251 